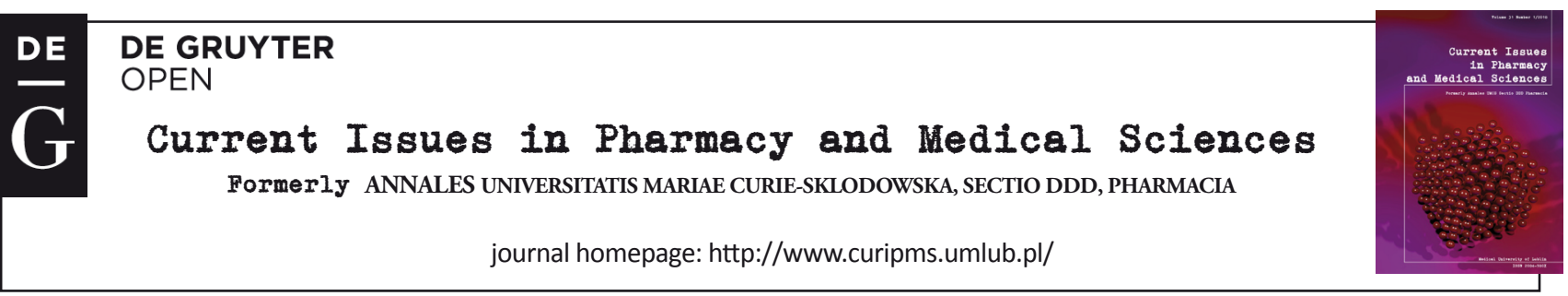

\title{
Prevalence and antibiotic susceptibility pattern of extended spectrum beta lactamases producing Escherichia coli isolated from outpatients with Urinary Tract Infections in Babol, Northern of Iran
}

\author{
Mana Baziboroun ${ }^{1}$, Masomeh Bayani ${ }^{1 \star}$, Zahra Poormontaseri ${ }^{2}$, \\ MeHran SHOKRI ${ }^{1}$, TAHMINEH BIAZAR ${ }^{1}$
}

${ }^{1}$ Infectious Diseases and Tropical Medicine Research Center, Health Research Institute, Babol University of Medical Sciences, Babol, I.R. Iran

${ }^{2}$ Department of infectious disease, Fasa University of Medical Science, Fars, Iran

\begin{tabular}{l}
\hline ARTICLE INFO \\
\hline Received 08 November 2017 \\
Accepted 15 February 2018
\end{tabular}

Keywords:

antibiotic resistance,

extended spectrum beta

lactamase,

Escherichia coli,

urinary tract infection.

\begin{abstract}
The incidence of community-acquired UTIs due to extended-spectrum beta-lactamase (ESBL) producing E. coli isolates, has increased worldwide and is considered a great problem in the treatment of infections. The aim of this study was to determine the prevalence of ESBL producing E. coli isolates in urine samples of outpatients in Babol, North of Iran. A total of 3699 urine samples from outpatients referred to Yahyanejad Hospital, Babol, Iran, were collected during 15 months, from March 2016 to June 2017. The samples were processed for bacterial culture using conventional methods, while antimicrobial susceptibility testing for $E$. coli isolates was performed by the disc diffusion method. The prevalence of ESBL producing E. coli isolates were assessed by Double-Disc tests. Of 3699 tested sample, 201 samples showed the growth of pathogens and among them, 106 isolates (52/7\%) were E. coli. The rate of ESBL producing E. coli isolates was $25 / 4 \%$. The majority $(81 / 5 \%)$ of the isolates were from females. High percent resistance was found against ceftazidime, cefotaxime, ceftriaxone, cefixime and ciprofloxacin, ranging from $61-100 \%$, while the highest percent susceptibility was seen to meropenem, piperacillin-tazobactam (100\%), followed by nitrofurantoin and amikacin (91\%). The high prevalence of ESBL producing E. coli isolates from outpatients calls for the need to aware of this increasing resistance between uropathogens and update bacterial susceptibility data and to set up our empirical therapy accordingly.
\end{abstract}

\section{INTRODUCTION}

Urinary Tract Infection (UTI) is one of the most common bacterial infections that are caused by gram negative bacteria. These can be Community-acquired or nosocomial $[1,2]$. More than $25 \%$ of all women experience some types of UTI at least once during their lifetime [3]. UTI is an absolute risk factor for bladder cancer and renal cell carcinoma. Indeed, clinicians often start empirical antimicrobial therapy before the culture results are available [4].

Escherichia coli (E. coli) is the most common cause of UTI, and nowadays we notice an increasing antibiotic resistance between $E$. coli associated infections worldwide. The well-spring of creating this resistance is the overuse of

\begin{tabular}{l} 
*Corresponding author \\
e-mail: m_baiany@yahoo.com \\
\hline
\end{tabular}

antibiotics. It is reported that urinary Tract isolates of $E$. coli from outpatient clinics have shown an increased resistance to certain antibiotics, including ampicillin and ciprofloxacin, rendering them near useless [5].

Beta lactams are the most frequent antibiotics that are prescribed by physicians. The main mechanism of resistance to these antibiotics, especially within gram negative bacteria, is the production of extended-spectrum beta-lactamases (ESBLs) [6,7]. These enzymes can completely hydrolize oximino-beta lactams such as the third generation cephalosporins. The majority of produced ESBL enzymes have been reported in E. coli and klebsiella pneumonia, but also are found in other entrobacteriacea [8,9]. ESBLs have a wide effect on penicillins and cephalosporins and aztreonam, but they do not effect carbapenems. ESBLs are prevented by 
beta-Lactamase inhibitors like clavulanic acid, tazobactam and sulbactam [9].Treatment of infections caused by ESBLproducing bacteria has become a world problem and has led to an increase in the death of patients, as well as a greater financial burden for treatment [10]. Because of rising antibiotic resistance between organisms and the high prevalence of ESBL producing E. coli in the community and its importance in managing UTIs, local hospital-based knowledge of the uropathogens and their antibiotic susceptibility pattern is necessary and helpful for physicians to choose appropriate empirical treatment $[11,12]$. Therefore, the goal of this study was to evaluate the prevalence of ESBL enzymes in E. coli isolates in outpatients with UTI.

\section{MATERIALS AND METHODS}

\section{Sample Collection and Bacteriology}

The present study is a cross-sectional study and was performed in shahid Yahyanejad Hospital, Babol, Iran from March 2016 to June 2017. Midstream specimens of urine from outpatients were obtained for semi-quantitative urine culture on blood agar and MacConkey agar by way of the standard loop method. After 24 hours incubation at $37^{\circ} \mathrm{C}$, colonies were counted, Samples with colonies of more than $10^{5}$ are considered as significant bacteriuria. E. coli isolates were identified with Gram-stains and biochemical tests containing oxidase, indole, methyl red, simmons citrate, lysine decarboxide, urease and glucose fermenting in TSI (Triple Sugar Iron) Agar medium and as described in standard bacteriological methods [13].

\section{Antibiotic susceptibility test}

Antibiotic sensitivity pattern to Clinical Laboratory Standard Institute (CLSI) guidelines was determined by the disc diffusion method on Muller-Hinton agar [14]. $E$. coli isolates were tested against common antibiotics used in the hospital: Amikacin (AMK $30 \mu \mathrm{gr}$ ), nitrofurantoin (NIT $30 \mu \mathrm{gr}$ ), ciprofloxacin (CIP $5 \mu \mathrm{gr}$ ), cefotaxime (CTX $30 \mu \mathrm{gr})$, ceftazidime (CAZ $30 \mu \mathrm{gr})$ meropenem (MEM $10 \mu \mathrm{gr})$, ceftriaxone (CRO $30 \mu \mathrm{gr})$, piperacillin-tazobactam (PTZ $100 \mu \mathrm{gr}$ ), ampicillin-sulbactam (SAM $20 \mu \mathrm{gr}$ ). All antimicrobial discs were obtained from MAST chemical co, England.

E. coli ATCC 25922 was used for quality control as CLSI recommendation.

\section{Detection of ESBLs production}

The ESBL-producing was determined by application of the Double-Disc (combined-disc) method. The bacterial suspension was prepared with $0 / 5$ Mcfarland dilution and was cultured on Muller-Hinton agar plates. Then, four discs containing ceftazidime $(30 \mu \mathrm{gr})$ and cefotaxime $(30 \mu \mathrm{gr})$ alone and in combination with $10 \mu \mathrm{gr}$ clavulanic acid (CAZ:30 $\mu \mathrm{gr} / \mathrm{CA} 10 \mu \mathrm{gr}$ ) and CTX:30 $\mu \mathrm{gr} / \mathrm{CA} 10 \mu \mathrm{gr}$ were placed on the agar surface. After incubation at $35 \pm 2{ }^{\circ} \mathrm{C}$ for $24 \mathrm{~h}, \mathrm{ESBL}$ production was evaluated. $\mathrm{A} \geq 5 \mathrm{~mm}$ increase in the diameter of inhibition zone produced by one combination disc versus its zone when tested alone, was considered as indicative of ESBL production. Klebsiella ATCC 700603 was used for quality control as recommended by CLSI.

\section{Data analysis}

All data were analyzed using SPSS software, version 16. The findings were presented as descriptive statistics regarding relative frequency.

\section{RESULTS}

During 15 months, a total of 3699 urine samples were obtained. Herein, 201 samples showed growth of pathogens; among them, E. coli was the most prevalent isolate $(52 / 7 \%)$. The majority $(81 / 5 \%)$ of the isolates were from females, while the remaining were from males $(18 / 5 \%)$. Figure 1 shows the age distribution of the patients with ESBL producing E. coli. The mean age of patients was $46 / 6$ years with a range of 5-81 years. More cases were recorded among the 40-60 year-olds (40/74\%).

ESBL positivity was detected in 27 of $106(25 / 4 \%)$ E. coli isolates. Figure 2 shows the susceptibility percentages of ESBL producing E. coli isolates to ten selected Antibiotics. Meropenem and piperacillin-tazobactam indicated highest percent susceptibility (100\%) to E. coli isolates, followed by amikacin (91\%), nitrofurantoin (91\%) and ampicillin-sulbac$\operatorname{tam}(75 \%)$. The rates of resistance in ESBL producing E. coli isolates are shown in figure 3 . The maximum resistance was seen against ceftazidime $(100 \%)$, cefotaxime $(89 \%)$, ceftriaxone (78\%), cefixime (67\%) and ciprofloxacin (61\%). ESBL producing $E$. coli isolates were divided into 6 groups according to their resistance patterns (Table 1).

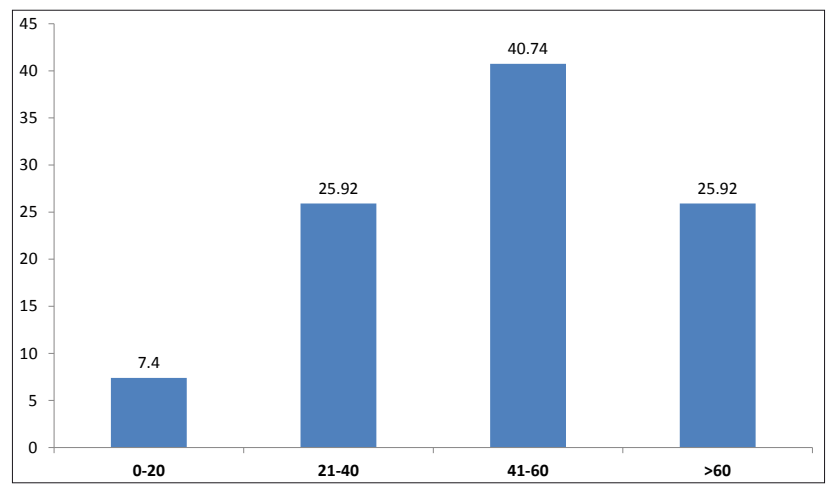

Figure 1. Age distribution of the patients with ESBL producing E. coli isolates

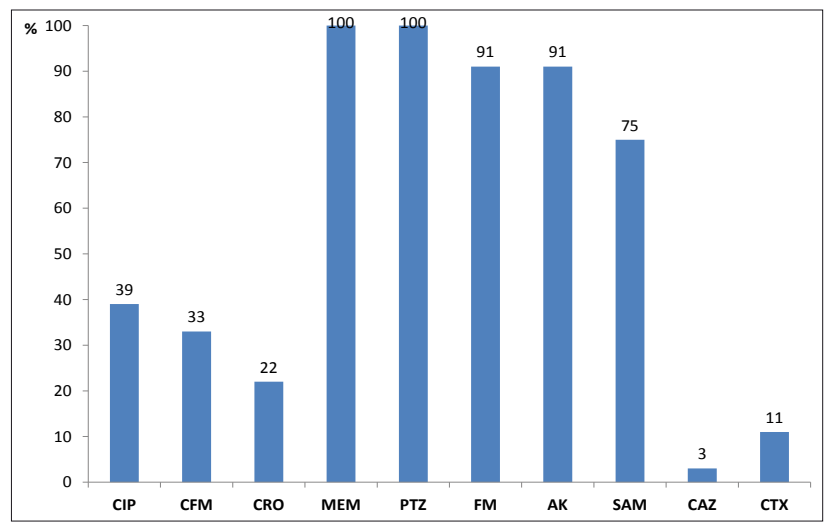

Figure 2. Percentages of antimicrobial susceptibility of ESBL producing E. coli isolates

Cyprofloxacin (CIP), Cefixime (CFM), Ceftriaxon (CRO), Meropenem (MEM), Piperacillin-tazobactam (PTZ), Nitrofurantoin (FM), Amikacin (AK), Ampicillin-sulbactam (SAM), Ceftazidime (CAZ), Cefotaxime (CTX) 


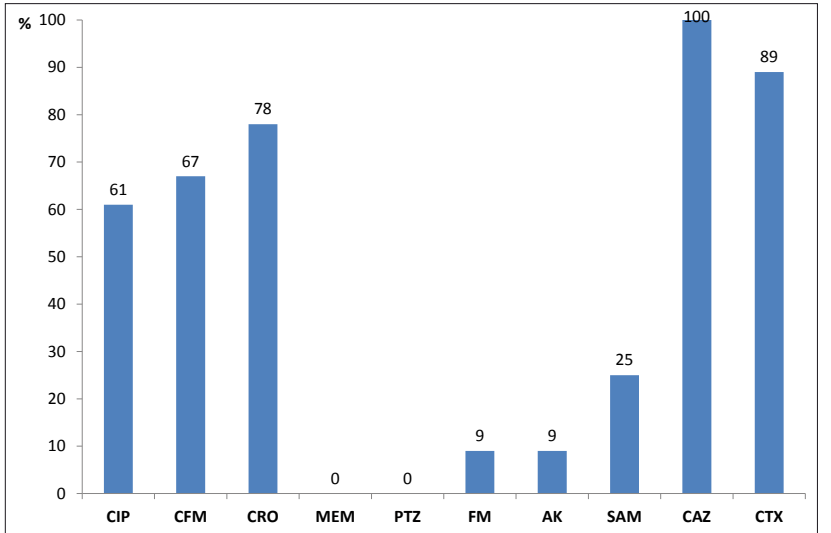

Cyprofloxacin (CIP), Cefixime (CFM), Ceftriaxon (CRO), Meropenem (MEM), Piperacillin-tazobactam (PTZ), Nitrofurantoin (FM), Amikacin (AK) Ampicillin-sulbactam (SAM), Ceftazidime (CAZ), Cefotaxime (CTX)

Figure 3. Antibiotic resistance pattern in ESBL producing E. coli isolates

Table 1. Resistance pattern of ESBL producing E. coli isolates

\begin{tabular}{|c|l|c|}
\hline Pattern & Resistance Pattern & $\begin{array}{c}\text { Percentage and number } \\
\text { of isolates }\end{array}$ \\
\hline Pattern 1 & Resistant to one drug & $18 / 5(5)$ \\
\hline Pattern 2 & Resistant to two drug & $29 / 6(8)$ \\
\hline Pattern 3 & Resistant to three drug & $22 / 2(6)$ \\
\hline Pattern 4 & Resistant to four drug & $22 / 2(6)$ \\
\hline Pattern 5 & Resistant to five drug & $3 / 7(1)$ \\
\hline Pattern 6 & Sensitive to all & $3 / 7(1)$ \\
\hline
\end{tabular}

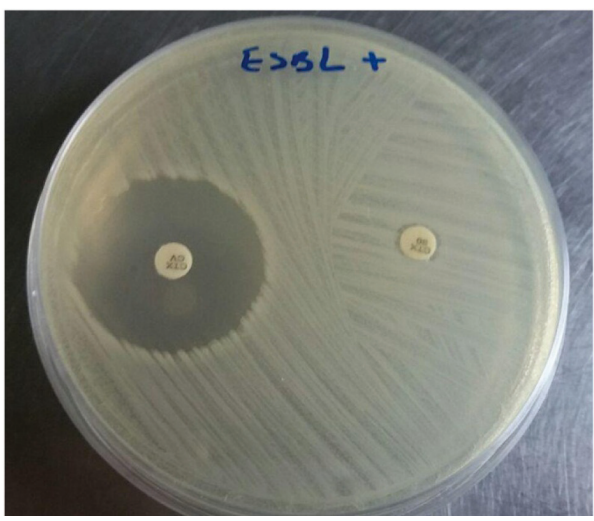

Figure 4. Plate showing ESBL production by Double-Disc method

\section{DISCUSSION}

UTIs caused by antibiotic-resistant bacteria are a major problem due to limited choices of treatment. Today, ESBLproducing $E$. coli isolates are the most common pathogens in community-acquired UTIs $[15,16]$. According to the WHO reports, Iran is one of the countries with the highest antibiotic consumption in the world, but in the global statistics, there is no report of antibiotic use in Iran [17]. In the current study, we evaluated the prevalence of ESBL producing $E$. coli isolates and their antibiotic susceptibility pattern in patients with community acquired UTI.

According to a report by Shakya et al., in Nepal, of 2014, similar to our study, the majority of ESBL producing $E$. coli isolates was from females $(81 / 8 \%)$, as was an Indian study in $2006(66 / 7 \%)$ and Paris in $2015(81 / 4 \%)$. Hence, women have a higher prevalence of UTI than men, mainly due to anatomic factors [18-21].

In a large number of studies from different parts of Iran, the prevalence of ESBL positivity UTIs were similar to our study (25/4\%), including that from Isfahan in 2012, Kermanshah in 2011, children in North of Iran in 2014, Iran as a whole in 2010 [10, 20,22,23] and also from some countries. In the last, the results were consistent to our study [24-26]. The rate of ESBL producing E. coli isolates in Nepal, Paris, Karachi, central India and Shiraz were 91/7\%, $69 / 4 \%, 54 \%, 41 / 6 \%$ and 69/2\%, respectively, which are high as compared to our results. In contrast, according to a Brazilian report of 2013 , the figure was $7 / 1 \%$ which is lower than the results of our study $[18,21,27-30]$. Contrary to our study, in Isfahan in 2012, E. coli isolates showed lower resistance against ceftazidime (32\%), cefotaxime (38\%) and ciprofloxacin $(33 / 4 \%)$, while in Shiraz, resistant to ciprofloxacin and ceftazidim was $38 / 5 \%$ and $63 / 5 \%$, respectively [20,29]. In Nepal in 2014, Turkey in 2016 and Korea in 2017, resistant to ciprofloxacin, cefotaxime and ceftazidim were also reported lower than our study $[18,25,33]$. However, ciprofloxacin resistance in Turkish isolates in 2010, in Paris, Saudi Arabia and Jaipur were reported as 84\%, 80\%, 88/9\% and $74 / 75 \%$ respectively, which are high as compared to our study $[16,21,24,26]$.

In a large number of studies, consistent with the results of recent study, high percent susceptibility was found to carbapenems, aminoglycosides and nitrofurantoin $[18,20,22,25,29,33]$ and also in agreement to our results, in Isfahan, Nepal and korea sensitivity to meropenem was reported $100 \%$ [18,20,33]. In Jaipur and Korea, susceptibility to piperacillin-tazobactam was high and similar to our study [26,33]. in India, isolates in 2006 showed highest resistance against nitrofurantoin (80\%) [19]. This effect is probability due to widely prescriptions of nitrofurantoin by physicians in the treatment of community-acquired UTI in this region.

Compared to advanced countries, in practice, the use of antibiotic guidelines is not common in Iran and, in fact, there are no local guidelines. The most inconsistent pattern of application has been seen in the prescription of ciprofloxacin and ceftazidim and cefotaxime [17,31]. High percent resistance exists against these antibiotics, probability duo to excessive use in recent years. In some studies, ciprofloxacin is the most prescribed antibiotic for treatment of UTI in Iran $[17,32]$. Thus, knowledge of the local susceptibility pattern of the more frequent UTIpathogens is necessary for choosing appropriate empirical therapy [15], and the results of our study can help us to choose more appropriate antibiotics in UTI treatment.

\section{CONCLUSION}

The results of our study showed a high prevalence of ESBL-producing E. coli isolates in outpatients. Because of too low resistance to nitrofurantoin, this antibiotic be prescribed in uncomplicated UTIs, while among the parenteral drugs, carbapenems, aminoglycosides and piperacillintazobactam can be the choice for complicated UTIs. 
In the end, it seems it is necessary to detect and prevent the emergence of resistance and update bacterial susceptibility data for setting up our empirical therapy regime accordingly.

\section{ACKNOWLEDGMENTS}

Authors are grateful to all staffs of Shahid Yahyanejad Hospital, Babol, Iran, for their help and support.

\section{CONFLICT OF INTEREST:}

There is no conflict of interest.

\section{REFERENCES}

1. Mehta M, et al.: Prevalence and antibiotic susceptibility pattern of multi-drug resistant Escherichia coli isolates from urinary tract infection (UTI) patients. Int J Life Sci Pharm Res. 2;6:2012.

2. Gerasimovska V, Gerasimovska-Kitanovska B: Extended spectrum beta-lactamase (ESBL) strains of E. coli as a cause of urinary tract infections in hospitalized patients. Antimicrobial Resistance and Infection Control. 4;P121:2015.

3. Noor $\mathrm{N}$, et al.: Urinary tract infections associated with multidrug resistant enteric bacilli: characterization and genetical studies. Pak J Pharm Sci. 17;115:2004.

4. Dehbanipour R, et al.: High prevalence of multidrug-resistance uropathogenic Escherichia coli strains, Isfahan, Iran. Journal of Natural Science, Biology, And Medicine. 7;22:2016.

5. Jan N, et al.: Plasmid profile analysis of multidrug resistant $\mathrm{E}$. coli isolated from UTI patients of Nagpur City, India. Romanian Biotechnological Letters. 14;4635:2009.

6. Mansouri S, Abbasi S: Prevalence of multiple drug resistant clinical isolates of extended-spectrum beta-lactamase producing Enterobacteriaceae in Southeast Iran. Iranian Journal of Medical Sciences. 35;101:2015.

7. Mana Baziboron, et al:Extended Spectrum Beta Lactamase Producing Escherichia Coli and Klebsiella Isolates in Patients with Urinary Tract Infection and Their Antibiotic Susceptibility Pattern in Babol, Northern Iran. International Journal of Advanced Biotechnology and Research. 8;1451:2017

8. MH S, MK SY: The prevalence of extended-spectrum beta-lactamases and CTX-M-1 producing Escherichia coli in urine samples collected at Tabriz city Hospitals. Tehran University Medical Journal TUMS Publications. 69;273:2011.

9. Ghafourian $\mathrm{S}$, et al.: Incidence of extended-spectrum beta-lactamaseproducing Klebsiella pneumoniae in patients with urinary tract infection. Sao Paulo Medical Journal. 130;37:2012.

10. Mohajeri P, et al.: Assessment of the frequency of Extended Spectrum Beta Lactamases Producing Escherichia coli Isolated from Urinary Tract Infections and its Antibiotic Resistance Pattern in Kermanshah. Journal of Ardabil University of Medical Sciences. 11;86:2011.

11. Hasan AS, et al.: Resistance patterns of urinary isolates in a tertiary Indian hospital. J Ayub Med Coll Abbottabad. 19;39:2007.

12. Dalela G, et al.: Antibiotic resistance pattern in uropathogens at a tertiary care hospital at Jhalawar with special reference to Esbl, Ampc $\beta$-Lactamase and MRSA production. J Clin Diagn Res. 6;51:2012.

13. Tille P . China: Elsevir Health Sciences; 2013. Bailey \& Scott's Diagnistic Microbiology.

14. Hombach M, et al.: Standardisation of disk diffusion results for antibiotic susceptibility testing using the sirscan automated zone reader. BMC Microbiology. 13;225:2013.
15. Bader MS, et al.: An update on the management of urinary tract infections in the era of antimicrobial resistance. Postgraduate Medicine. 129;242:2017.

16. Azap Ö, et al.: Risk factors for extended-spectrum $\beta$-lactamase positivity in uropathogenic Escherichia coli isolated from community-acquired urinary tract infections. Clinical Microbiology and Infection. 16;147:2010.

17. Shiva Hashemi, et al.:Irrational antibiotic prescribing: a local issue or global concern? Excli Journal. 12;384:2013

18. Shakya P, et al.: ESBL Production Among E. coli and Klebsiella spp. Causing Urinary Tract Infection: A Hospital Based Study. The Open Microbiology Journal. 11;23:2017.

19. Akram M, et al.: Etiology and antibiotic resistance patterns of community-acquired urinary tract infections in JNMC Hospital Aligarh, India. Annals of Clinical Microbiology and Antimicrobials. 6;4:2007.

20. Moayednia R, et al.: Frequency assessment of $\beta$-lactamase enzymes in Escherichia coli and Klebsiella isolates in patients with urinary tract infection. Journal of research in medical sciences: the official journal of Isfahan University of Medical Sciences. 19;S41:2014.

21. D. Chervet, et al.:Antimicrobial resistance in community-acquired urinary tract infections in Paris. Medecine et Maladies Infectieuses. 2017.

22. Pourakbari B, et al.: Increase resistant rates and ESBL production between E. coli isolates causing urinary tract infection in young patients from Iran. Brazilian Journal of Microbiology. 43;766:2012.

23. Eslami G, et al.: Epidemiology of extended spectrum beta lactamases producing E. coli genes in strains isolated from children with urinary tract infection in north of Iran. Journal of Mazandaran University of Medical Sciences. 25;270:2016.

24. Fahad A, et al.: Incidence and molecolar characterization of the extended spectrum beta lactamase-producing Escherichia coli isolated from urinary tract infections in Eastern Saudi Arabia. Saudi Med J. 38;811:2017.

25. Nisel Yilmaz, et al.:Antimicrobial susceptibilities of Escherichia coli isolates as agents of community-acquired urinary tract infection (2008-2014). Turk J Urol. 42;32:2016.

26. Smita Sood and Ravi Gupta: Antibiotic Resistance Pattern of Community Acquired Uropathogens at a Tertiary Care Hospital in Jaipur, Rajasthan. Indian J Community Med. 37;39:2012.

27. Hassan SA, et al.: Occurrence of multidrug resistant and ESBL producing E. coli causing urinary tract infections. J Basic Appl Sci. 7;39:2011.

28. Trupti Bajpai, et al.: Prevalence of extended spectrum beta-lactamase producing uropathogens and their antibiotic resistance profile in patients visiting a tertiary care hospital in central India: Implications on empirical therapy. Indian Journal of Pathology and Microbiology. 57;407:2014.

29. Pouladfar G, et al.: The antibiotic susceptibility patterns of uropathogens among children with urinary tract infection in Shiraz. Medicine. 96:2017.

30. Gonçalves LF, et al.: Multidrug resistance dissemination by extended-spectrum $\beta$-lactamase-producing Escherichia coli causing community-acquired urinary tract infection in the Central-Western Region, Brazil. Journal of global antimicrobial resistance. 6;1:2016.

31. Hossein Khalili, et al.: Adherence to Empirical Antibiotic Therapy Guideline in a Refferal Teaching Hospital, Tehran, Iran. Acta Medica Iranica. 50;47:2020.

32. Bahman Haghighi, et al.: Comparison of 3-day and 7-day ciprofloxacin regimen for the treatment of uncomplicated urinary tract infection in women: A randomized double-blind clinical trial. Iranian Journal of Clinical Infectious Diseases. 5;70:2010.

33. Jin Ju Park, et al.:Antimicrobial susceptibilities of Entrobacteriaceae in Community-Acquired Urinary Tract Infections during a 5-year Period: A Single Hospital Study in Korea. Infect Chemother. 49;184:2017. 